Artigo Original

Original Article

Maria Eduarda Braga de Araújo $0^{1}$ (1) Maria Clara de Oliveira Lima ${ }^{1}$ Wanderson Laerte de Oliveira Carvalho ${ }^{2}$ (D) Joseli Soares Brazorotto ${ }^{1}$ (D)

Descritores Reprodutibilidade dos Testes Audição Percepção Auditiva Perda Auditiva Reabilitação

Keywords Reproducibility of Tests Hearing Auditory Perception Hearing Loss Rehabilitation

Endereço para correspondência: Joseli Soares Brazorotto Avenida General Gustavo Cordeiro de Farias, s/n, Petrópolis, Natal (RN), Brasil. CEP: 59012-570.

E-mail: brazorotto@yahoo.com ou josisbraz@gmail.com

Recebido em: Novembro 12, 2019

\section{Adaptação do protocolo Indicadores de Performance Funcional Auditiva Brasileiro - Versão Reduzida}

\author{
Adaptation of the Brazilian Functional Auditory \\ Performance Indicators - Short Version
}

\begin{abstract}
RESUMO
Objetivo: Adaptar o protocolo Indicadores de Performance Funcional Auditiva Brasileiro para uma versão reduzida, incluindo a produção de material e de um manual de aplicação. Método: Estudo do tipo metodológico, descritivo e transversal, com abordagem quantiqualitativa, realizado com sete fonoaudiólogos que aplicaram o protocolo em 34 crianças com deficiência auditiva e seus responsáveis. Cumpriram-se a análise da validade de conteúdo, bem como da confiabilidade do instrumento por meio da avaliação da equivalência semântica, da análise da satisfação dos fonoaudiólogos e dos testes estatísticos alfa de Cronbach e coeficiente de Kappa. Resultados: Foi adaptada a versão reduzida denominada FAPI-r, com coeficientes que indicaram consistência interna e concordância interavaliadores quase perfeitos. Os fonoaudiólogos referiram satisfação com o instrumento e relataram que o manual de aplicação facilitou o entendimento sobre a aplicação do teste. Observou-se, no entanto, a não concordância entre a avaliação dos fonoaudiólogos e as respostas das famílias, indicando a necessidade de maior inserção destas no cenário terapêutico. Conclusão: Realizada a adaptação do FAPI-r, constatando-se sua validade e confiabilidade, com futuros desdobramentos para a pesquisa e atuação clínica na população de crianças com deficiência auditiva.
\end{abstract}

\begin{abstract}
Purpose: To adapt the Brazilian Functional Auditory Performance Indicators protocol to a short version including the production of material and an application manual. Methods: Methodological, descriptive, cross-sectional study with a quantitative and qualitative approach conducted with seven speech-language therapists who applied the protocol to 34 children with hearing loss and their guardians. The analysis of content validity and the instrument reliability evaluated the semantic equivalence, analysis of satisfaction of speech therapists, and statistical tests of Cronbach's Alpha and Kappa coefficient. Results: The short version, called FAPI-r, was adapted considering coefficients that indicate internal consistency and almost perfect inter-evaluator agreement. Speech-language therapists reported satisfaction with the instrument and stated that the manual facilitates the understanding of the test application. However, there was no agreement between the assessment of speech-language therapists and the answers of families, indicating a need for a greater insertion of families in the therapeutic scenario. Conclusion: The adapted FAPI-r has validity and reliability, promising future developments for research and clinical performance in the population of hard of hearing children.
\end{abstract}

Trabalho realizado na Universidade Federal do Rio Grande do Norte - UFRN - Natal (RN), Brasil.

${ }^{1}$ Departamento de Fonoaudiologia, Laboratório de Inovação Tecnológica em Saúde- LAIS, Hospital Universitário Onofre Lopes - HUOL, Universidade Federal do Rio Grande do Norte - UFRN - Natal (RN), Brasil.

${ }^{2}$ Departamento de Matemática e Estatística, Universidade do Estado do Rio Grande do Norte - UERN - Mossoró $(\mathrm{RN})$, Brasil.

Fonte de financiamento: nada a declarar.

Conflito de interesses: nada a declarar. 


\section{INTRODUÇÃO}

O impacto da deficiência auditiva na infância está amplamente documentado, bem como o papel das habilidades auditivas para o desenvolvimento infantil ${ }^{(1,2)}$.

Considerando que o avanço das habilidades auditivas na criança com deficiência auditiva tem impacto em seu desenvolvimento global, é imprescindível que o fonoaudiólogo utilize ferramentas que o auxiliem a mensurar o progresso auditivo esperado para cada caso, realizando a análise individual dos fatores positivos ou eventuais dificultadores da evolução das crianças ${ }^{(3-8)}$.

Somado a isso, a utilização de avaliações completas e padronizadas do progresso auditivo nesta população permite o acompanhamento da evolução terapêutica, a comparação entre grupos, a reavaliação do programa de (re)habilitação auditiva, a comparação multicêntrica, assim como sinaliza para os profissionais e para a família quando há algum desvio de aquisição, o que viabiliza aperfeiçoar habilidades que são esperadas para a idade e para a escolaridade das crianças ${ }^{(9-11)}$.

O inventário Functional Auditory Performance Indicators (FAPI) foi desenvolvido nos Estados Unidos da América (EUA) e sua utilização como instrumento de avaliação de crianças com deficiência auditiva é preconizada pela American Speech-LanguageHearing Association (ASHA). Diante da escassez de protocolos traduzidos e adaptados para o português brasileiro que avaliem todas as habilidades auditivas em um mesmo instrumento, o FAPI, em sua versão original, foi traduzido e adaptado, dando origem ao instrumento Indicadores de Performance Funcional Auditiva Brasileiro (FAPI brasileiro) ${ }^{(9)}$; que foi resumido e validado, culminando na versão reduzida aqui apresentada (Anexo 1).

Outros protocolos foram traduzidos e adaptados para o português brasileiro e têm sido utilizados em serviços de saúde auditiva ${ }^{(9,10,12-15)}$, no entanto, o FAPI brasileiro destaca-se como a ferramenta mais completa recentemente publicada em português, visto que avalia sete categorias de habilidades auditivas em diferentes condições de escuta, contando com informações da família e/ou dos professores, para além da observação realizada clinicamente, o que permite ao fonoaudiólogo traçar um perfil bastante realista de desenvolvimento das habilidades auditivas de crianças com deficiência auditiva ${ }^{(9)}$, o que contribui sobremaneira para o planejamento terapêutico e orientações ofertadas aos familiares destas crianças.

O FAPI possui padrões de normalidade até os cinco anos de idade. Dentre as categorias de habilidades auditivas analisadas, estão: consciência sonora e sons significativos, feedback auditivo e integração, localização da fonte sonora, discriminação auditiva e reconhecimento, compreensão auditiva, memória de curto prazo e processamento auditivo linguístico; em condições de escuta fáceis (estímulos auditivos emparelhados com sinais visuais, ambiente silencioso, estímulos apresentados próximos à criança e respostas solicitadas) e difíceis (estímulos somente auditivos, distantes, ambientes ruidosos e respostas espontâneas). Dessa forma, o protocolo visa a identificação dos pontos fortes e fracos de cada criança, com o intuito de otimizar o processo de (re)habilitação auditiva ${ }^{(9)}$.

Embora o FAPI seja considerado um protocolo bastante completo, a quantidade de itens avaliados pode dificultar a aplicabilidade clínica do instrumento. Dessa forma, justifica-se a elaboração de um protocolo em versão reduzida, trazendo maior viabilidade de utilização desta medida na rotina de acompanhamento da (re)habilitação auditiva. Além disso, observou-se a necessidade de esclarecer quais os materiais que devem ser utilizados para avaliar cada habilidade auditiva proposta e a maneira de fazer as solicitações à criança, minimizando, assim, o possível viés de variabilidade de aplicação.

É válido frisar que, mesmo que se recomende a aplicação do FAPI para crianças até os cinco anos de idade, é possível que crianças mais velhas ainda estejam em evolução da percepção auditiva, acarretando, assim, a necessidade de uma ferramenta que permita traçar seu progresso no processo terapêutico.

Destaca-se a necessidade de instrumentos de avaliação reduzidos que se proponham a medir os mesmos constructos que as versões mais extensas ${ }^{(16)}$, pois, ao adaptar um instrumento, $\mathrm{o}$ pesquisador é capaz de comparar os dados obtidos em diferentes amostras, de contextos diversos, permitindo uma maior equidade na avaliação, uma vez que se trata de uma mesma medida, que avalia o constructo a partir de uma mesma perspectiva teórica e metodológica ${ }^{(16)}$

Esta pesquisa para a adaptação da versão reduzida do FAPI (FAPI-r) contou com a análise da validade de conteúdo e da confiabilidade (consistência interna ou reprodutibilidade) do protocolo FAPI-r $\mathrm{r}^{(17-19)}$.

Isto posto, dada a necessidade de uma ferramenta que seja de aplicação breve e que traga informações sobre o perfil de desenvolvimento de todas as habilidades auditivas pela criança com deficiência auditiva, esta pesquisa teve como objetivo adaptar o protocolo FAPI brasileiro, contando com as análises de validade de conteúdo e da confiabilidade da versão reduzida (FAPI-r), incluindo a elaboração de um manual de aplicação, a fim de otimizar a utilização do instrumento na prática clínica, com vistas a aprimorar o acompanhamento do desenvolvimento das habilidades auditivas em crianças com deficiência auditiva.

\section{MÉTODO}

Trata-se de um estudo do tipo metodológico, descritivo e transversal, com abordagem quantiqualitativa, cuja execução foi aprovada pelo Comitê de Ética da instituição responsável, sob parecer de número 1.144.295.

O estudo foi realizado em um serviço de saúde auditiva de alta complexidade do Sistema Único de Saúde, de natureza filantrópica.

Os fonoaudiólogos participantes da pesquisa, bem como as famílias das crianças com deficiência auditiva, foram esclarecidos sobre o estudo e aceitaram participar, assinando o Termo de Consentimento Livre e Esclarecido (TCLE).

A amostra foi selecionada por conveniência, sendo composta pelas sete fonoaudiólogas atuantes no setor de (re)habilitação auditiva e por 34 crianças com deficiência auditiva de graus moderado a profundo, sem faixa etária previamente definida, e suas famílias.

Foram critérios de inclusão: crianças com deficiência auditiva usuárias de dispositivos auxiliares à audição (AASI e/ou IC), com frequência mínima de $75 \%$ à terapia fonoaudiológica no 
serviço de (re)habilitação auditiva participante do estudo. Não foram excluídas da pesquisa crianças com outras necessidades associadas à deficiência auditiva. Vale informar que 5 crianças foram, durante a coleta de dados, diagnosticadas com outras necessidades, o que, de acordo com as fonoaudiólogas participantes, não impediu a aplicação do protocolo, sendo, portanto, seus resultados considerados na análise geral deste estudo.

As famílias apresentaram perfil de renda classificado como nível socioeconômico baixo e a escolaridade média dos familiares respondentes foi o ensino fundamental. As fonoaudiólogas participantes tinham experiência mínima de 1 ano no setor de (re)habilitação auditiva da instituição.

O instrumento $\mathrm{FAPI}^{(9)}$, objeto de estudo em questão, é composto por 61 itens, organizados em 33 seções, e consta de um formulário para o preenchimento pelo fonoaudiólogo, em momento de avaliação clínica com a criança, além de questionamentos realizados às famílias.

O protocolo adaptado, Indicadores de Performance Funcional Auditiva Versão Reduzida - FAPI-r e seu referido manual, foi composto por dois formulários um para o fonoaudiólogo, constando de 25 itens, e outro para a família com 15 itens, ambos organizados em 15 seções, divididos nas mesmas sete categorias de habilidades auditivas propostas pelo instrumento original.

Para a consecução do objetivo da pesquisa, a coleta de dados, com as crianças e suas famílias pelas fonoaudiólogas, foi precedida pela adaptação do instrumento de sua versão completa traduzida para o português brasileiro para a versão reduzida e contou com as seguintes etapas:

1) Análise da Validade de Conteúdo:

Etapa 1a): realizou-se a análise pormenorizada do instrumento em sua versão original: a pesquisadora e duas especialistas analisaram o instrumento FAPI brasileiro ${ }^{(9)}$ e identificaram, dentre os itens das habilidades auditivas avaliadas, quais seriam os mais representativos e que pudessem ser aplicados em tempo reduzido, além de analisarem a forma de pontuação proposta no protocolo original;

Etapa 1b): construção do instrumento na versão reduzida: foi elaborada a versão reduzida e realizada uma nova análise pela pesquisadora e por duas especialistas, contando com a revisão da equivalência semântica de cada item em relação ao protocolo original. Também foi realizada nesta etapa a seleção e produção dos materiais (figuras, textos e outros materiais) que seriam utilizados para a aplicação do protocolo em versão reduzida, bem como foi feita, em cabina acústica, a gravação dos itens que foram apresentados em situação de mensagem gravada;

Etapa 1c): estudo-piloto I: aplicação prévia do instrumento com 2 crianças ouvintes, um menino de três anos e uma menina de cinco anos de idade, no intuito de se avaliar a compreensão das crianças em relação às tarefas solicitadas, medir o tempo de aplicação do teste e analisar se as habilidades auditivas-alvo avaliadas seriam medidas na versão reduzida. Após a aplicação nas crianças ouvintes, foram observados os itens a serem reescritos para melhor entendimento da instrução pelas crianças, bem como foi realizado o ajuste de figuras utilizadas e do número de estímulos, para otimizar a compreensão do protocolo, resultando em sua melhor aplicabilidade;

Etapa 1d): realizada a revisão dos escores da pontuação, sendo que o escore total para a versão reduzida variou de $0-35 \%$ (não presente - NP), 36-79\% (em processo - EP) e $80-100 \%$ (adquirido -A). Vale registrar que foi denominada de "não presente" até $35 \%$ e não até $10 \%$, como é proposto nas versões original e brasileira, considerando a redução do número de itens avaliados e a possibilidade de observação de acertos aleatórios, sensibilizando o ponto de corte do instrumento para as habilidades que não são demonstradas claramente pela criança durante a avaliação, o que alerta o avaliador para uma análise mais pormenorizada destes itens durante o acompanhamento ou processo terapêutico. Além disso, separou-se o protocolo em dois formulários: o da família e o do avaliador, com o intuito de facilitar a aplicação. Para tornar o instrumento mais claro, foi suprimida a categoria de pontuação "emergente", pois observou-se certa dificuldade de interpretar, em uma sessão de avaliação, se a habilidade auditiva estaria emergindo ou se já estaria em processo de desenvolvimento;

Etapa 1e): aplicação-piloto do teste em 1 criança com deficiência auditiva para checagem das adaptações realizadas na versão reduzida, em especial, quanto à compreensão do teste pela criança ${ }^{(16)}$;

Etapa 1f): revisão final do protocolo de teste e elaboração do manual de aplicação, pontuação e interpretação, entregue aos fonoaudiólogos do serviço de (re)habilitação auditiva participante.

\section{2) Análise da Confiabilidade:}

Etapa 2a): as fonoaudiólogas do serviço de (re)habilitação auditiva receberam um kit contendo o manual do FAPI-r, formulários de aplicação e pontuação, além dos materiais audiovisuais para a aplicação do teste (figuras e estímulos gravados) e foram orientadas sobre os objetivos do teste e sobre sua aplicação. Aplicaram o protocolo com as crianças atendidas em terapia por cada uma delas, com faixa etária entre 1 ano e 3 meses até 13 anos e 7 meses, com a média de idade de 8 anos e 8 meses. Além disto, um instrumento de análise sobre a aplicabilidade do FAPI-r na rotina clínica e sobre a satisfação das fonoaudiólogas com ele foi preenchido por elas. Tais dados foram analisados estatisticamente por meio do coeficiente de Kappa para o estabelecimento da concordância interavaliadores;

Etapa 2b): análise estatística da consistência interna do instrumento por meio do cálculo do alfa de Cronbach, tanto para o formulário clínico como para o questionário aplicado à família. Além disso, mediu-se a concordância interavaliadores entre os resultados da avaliação das fonoaudiólogas e as respostas das famílias acerca do desenvolvimento das habilidades auditivas das crianças avaliadas, por meio do coeficiente de Kappa. 


\section{RESULTADOS}

Os resultados serão apresentados de acordo com a sequência da adaptação do protocolo.

Quanto à análise da validade de conteúdo, houve a redução de 61 itens para 25 itens, e quanto às seções, de 33 para 15 , considerando-se as habilidades auditivas mais representativas dentro de cada categoria de habilidades auditivas; mantiveramse os sete domínios das habilidades auditivas do instrumento original.

O manual de aplicação, as instruções às crianças em mensagem gravada, bem como as figuras foram elaborados e foi realizada a análise da equivalência semântica, com a indicação de boa aplicabilidade, a partir dos estudos-pilotos realizados com as crianças ouvintes e com a criança com deficiência auditiva.

Sobre a elaboração do manual de aplicação, pontuação e interpretação dos resultados, este seguiu as orientações do protocolo original e foi ampliado, contando com: a forma de instrução à criança e uso dos materiais de aplicação, a maneira de se realizar a pontuação e a interpretação dos resultados.
Quanto à análise da confiabilidade (consistência interna) do instrumento, obteve-se o coeficiente alfa de Cronbach equivalente a 0,9355 para o protocolo aplicado pelas fonoaudiólogas e de 0,8065 para o questionário respondido pelas famílias. Dessa forma, foi atingido o critério de alfa $\geq 0,80$, indicando consistência interna quase perfeita da versão reduzida.

Em relação à análise da concordância entre o julgamento das fonoaudiólogas sobre o instrumento, o coeficiente de kappa foi igual a 1, que corresponde a uma concordância quase perfeita (kappa entre 0,81 e 1).

A Figura 1 demonstra os resultados do questionário de satisfação preenchido pelas fonoaudiólogas.

Quando questionadas se o FAPI-r é capaz de avaliar as habilidades propostas, todas as profissionais (100\%) responderam que "sim"; além de concordarem que o instrumento é viável para utilização na prática clínica.

A Tabela 1 apresenta os resultados da aplicação do coeficiente de Kappa para a comparação entre a avaliação realizada pelas fonoaudiólogas e as respostas das famílias.

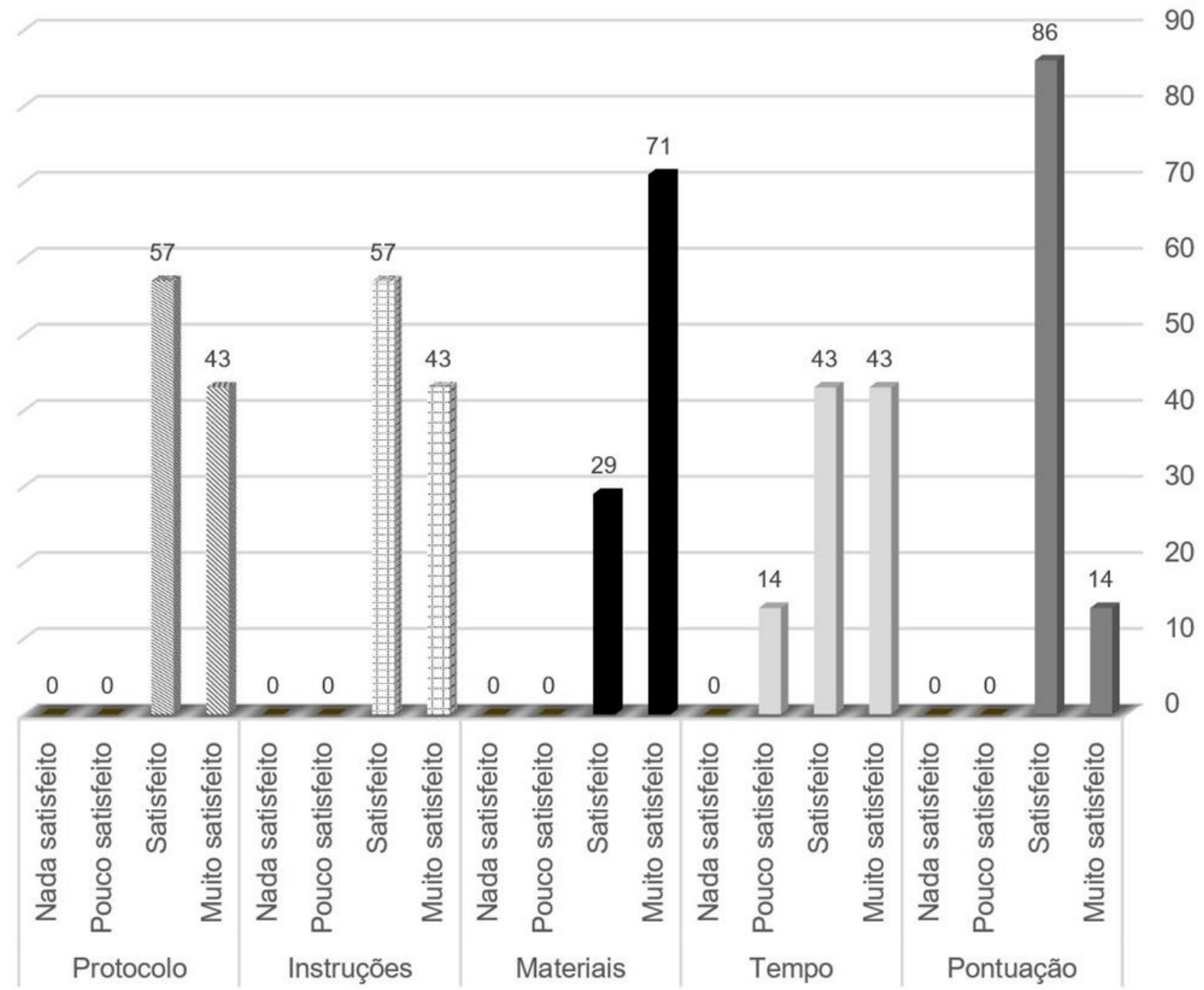

Figura 1. Nível de satisfação das fonoaudiólogas com o FAPI-r 
Tabela 1. Determinação da concordância interavaliadores pela aplicação do coeficiente de Kappa aos resultados da avaliação das fonoaudiólogas e às respostas das famílias.

\begin{tabular}{cc}
\hline Categorias de habilidade auditivas & $\begin{array}{c}\text { kappa } \\
\text { comparativos }\end{array}$ \\
\hline Consciência sonora e sons significativos/ localização & 0,6 \\
Feedback auditivo e integração & 0,28 \\
Discriminação auditiva e reconhecimento & 0,44 \\
Compreensão auditiva & 0,2 \\
Memória auditiva de curto prazo & 0,2 \\
Processamento auditivo linguístico & $-0,28$ \\
\hline
\end{tabular}

Nota: Kappa menor que 0 - insignificante, Kappa entre 0 e 0,2 - fraco, Kappa entre 0,21 e 0,40 - razoável, Kappa entre 0,41 e 0,60 - moderado, Kappa entre 0,61 e 0,80 - forte, Kappa entre 0,81 e 1 - quase perfeito

\section{DISCUSSÃO}

O acompanhamento do desenvolvimento das habilidades auditivas na criança com deficiência auditiva é fundamental e destacado na literatura para o melhor gerenciamento terapêutico ${ }^{(3-8)}$.

Instrumentos que possam mensurar de modo global e em ampla faixa etária tal evolução são necessários, especialmente na realidade brasileira ${ }^{(9)}$, proposta da referida pesquisa, que obteve resultados promissores considerando a validade de conteúdo e confiabilidade quanto à aplicabilidade do instrumento ora adaptado, denominado FAPI-r.

A partir das etapas de adaptação de instrumentos seguidas neste estudo, observou-se concordância com a literatura específica sobre a adaptação de protocolos ou testes clínicos que destacam a necessidade de várias etapas para que possa um instrumento ser utilizado por diferentes profissionais e em diferentes contextos, sendo capaz de produzir resultados válidos e confiáveis. Para que os protocolos consigam atingir esses objetivos, é necessário que possuam propriedades psicométricas adequadas, dentre as quais se destacam a validade e a confiabilidade ${ }^{(17-20)}$.

Os parâmetros analisados referentes à validade de conteúdo e confiabilidade atestaram a aplicabilidade clínica do FAPI-r e sua alta consistência interna ${ }^{(16)}$.

Ainda que já existam instrumentos para a avaliação clínica das habilidades auditivas na população infantil, a maioria dos protocolos disponíveis em português correspondem a adaptações transculturais ${ }^{(9,10,12-15)}$, e não foi observada, na literatura, a continuidade das pesquisas que buscassem analisar as suas propriedades psicométricas, sendo uma necessidade a elaboração e/ou a adaptação de protocolos que cumpram o rigor metodológico preconizado.

Além da adaptação do protocolo de aplicação, constatou-se a necessidade de adaptar o manual do FAPI brasileiro, a partir da verificação dos dois especialistas e da pesquisadora na etapa de análise da validade de conteúdo. Esta adaptação referiu-se a explicações detalhadas sobre como realizar as solicitações às crianças, bem como a respeito de quais os materiais que deveriam ser utilizados em cada seção proposta pelo instrumento FAPI-r. A elaboração de manuais de aplicação visa facilitar o entendimento dos avaliadores, sistematizar os procedimentos e diminuir a probabilidade de erros na avaliação clínica e na interpretação dos resultados ${ }^{(9)}$, fato observado a partir das respostas das fonoaudiólogas participantes, que referiram a utilidade do manual elaborado.

A estimativa do tempo de aplicação dos instrumentos de avaliação é importante, visto que, os resultados da (re)habilitação na deficiência auditiva, podem ser empregados como indicadores de qualidade de serviços de saúde auditiva. Dessa forma, é imprescindível a utilização de instrumentos rápidos e possíveis de serem aplicados no sistema ambulatorial. O FAPI brasileiro necessitou de mais de uma sessão para ser aplicado ${ }^{(9)}$, enquanto a média de tempo mensurado pelas fonoaudiólogas para a avaliação clínica e aplicação do questionário com as famílias com o FAPI-r foi de no máximo 40 minutos, sendo que este tempo de aplicação ainda pode ser diminuído considerando a maior experiência do avaliador, a idade da criança e o seu progresso das habilidades auditivas. Ressalta-se que as fonoaudiólogas referiram que, após algumas sessões em que se familiarizaram com o protocolo, o tempo de aplicação foi reduzido.

Quanto ao questionário aplicado com as famílias das crianças com deficiência auditiva, observou-se que elas julgaram, em geral, que as crianças tinham um progresso das habilidades auditivas menor do que o avaliado clinicamente, demonstrado pela pouca concordância observada na análise de Kappa para as habilidades analisadas no FAPI-r.

Verificou-se ainda que os familiares tiveram dificuldade em identificar, em especial, o progresso de seus filhos nas habilidades auditivas mais avançadas, fato este que explica a menor concordância expressada pelo índice de kappa para tais habilidades, a saber: compreensão auditiva, memória de curto prazo e processamento auditivo linguístico. A habilidade auditiva que apresentou o maior coeficiente de concordância $(0,60)$, classificado como moderado, foi a consciência sonora de sons significativos/localização, que é a primeira habilidade a ser adquirida na sequência gradativa de desenvolvimento das habilidades auditivas. A menor concordância, classificada como insignificante, foi atribuída ao processamento auditivo linguístico $(-0,28)$.

Notou-se, pois, que, para os familiares, as habilidades auditivas elementares são mais facilmente observadas em sua rotina com as crianças, contrastando com as habilidades mais avançadas, visto que os responsáveis indicaram uma menor pontuação para seus filhos do que aquela obtida em situação de teste com o fonoaudiólogo. É importante destacar que, se o ambiente domiciliar não propicia situações de diálogo em que as habilidades de compreensão auditiva e processamento auditivo linguístico são requeridas, as famílias certamente terão dificuldade em observar as crianças exibindo tais habilidades, uma hipótese para os resultados observados no presente estudo.

É imprescindível, portanto, que as famílias sejam orientadas sobre o que são as habilidades auditivas, bem como sobre como tornar o ambiente domiciliar propício ao desenvolvimento delas. Assim, a participação efetiva da família na terapia fonoaudiológica, dando condições para que os familiares avaliem o desenvolvimento, mesmo que sutil, destas habilidades de seus filhos, permite aos cuidadores atuarem naturalmente para o progresso do desenvolvimento auditivo das crianças com deficiência auditiva ${ }^{(21)}$.

Ressalta-se que as crianças cujos responsáveis referiram pior desempenho auditivo foram as que tiveram maior tempo de privação sensorial e menor idade auditiva, o que demonstra que seus familiares 
também estão inseridos há menos tempo no processo terapêutico e ainda se familiarizando com o desenvolvimento das habilidades auditivas. Espera-se que, conforme sejam orientadas acerca das etapas do progresso auditivo, desenvolvam melhor percepção das potencialidades e das dificuldades dos seus filhos, atuando como agentes estimuladores dentro das necessidades das crianças ${ }^{(5,21,22)}$.

\section{CONCLUSÃO}

A versão reduzida adaptada, denominada FAPI-r, demonstrou aplicabilidade clínica e proporcionou, de forma breve, um perfil abrangente das habilidades auditivas de crianças com deficiência auditiva inseridas em um serviço de (re)habilitação auditiva, com validade de conteúdo e confiabilidade confirmadas pela análise estatística.

As fragilidades do estudo referiram-se, em especial, à sua característica local, bem como à necessidade de testar o FAPI-r em uma amostra mais representativa, com possibilidade de análises estatísticas complementares para o estabelecimento das validades de constructo e de critério do instrumento em sua versão reduzida, visando o fortalecimento da adaptação realizada.

Estudos multicêntricos com a aplicação do referido instrumento podem gerar dados importantes para futuras pesquisas na área e na melhoria do acompanhamento de crianças com deficiência auditiva em serviços especializados.

Conclui-se, pois, que o FAPI-r pode ser uma importante ferramenta para o acompanhamento clínico e o gerenciamento terapêutico fonoaudiológico, que otimize os benefícios da tecnologia para o desenvolvimento das crianças com deficiência auditiva.

\section{AGRADECIMENTOS}

Agradecemos ao Centro SUVAG do RN a constante parceria e a todas as fonoaudiólogas, familiares e crianças participantes deste estudo. Um agradecimento especial à fonoaudióloga Ingrid Rafaella Dantas dos Santos pelo auxílio durante a coleta dos dados.

\section{REFERÊNCIAS}

1. Bornstein $\mathrm{S}$. The importance of early identification and intervention for children with hearing loss. Part 1: human Development. J Health Commun. 2018;3:1-6.

2. Vavatzanidis NK, Mürbe D, Friederici AD, Hahne A. Establishing a mental lexicon with cochlear implants: an ERP study with young children. Sci Rep. 2018;8(1):910. http://dx.doi.org/10.1038/s41598-017-18852-3. PMid:29343736.

3. Bicas RS, Guijo LM, Delgado-Pinheiro EMC. Oral communication and auditory skills of hearing-impaired children and adolescents and the speech therapy rehabilitation process. Rev CEFAC. 2017;19(4):465-74. http:// dx.doi.org/10.1590/1982-0216201719412516.

4. Colalto CA, Goffi-Gomez MVS, Magalhães ATM, Samuel PA, Hoshino $\mathrm{ACH}$, Porto BL, et al. Vocabulário expressivo em crianças usuárias de implante coclear. Rev CEFAC. 2017;19(3):308-19. http://dx.doi. org/10.1590/1982-021620171937216.

5. Shekari E, Nakhshab M, Valinejad V, Modarres Zadeh A, Hosseinpour A. Review paper: a systematic review of the effectiveness of early intervention and the role of parents in language development of hearing loss children. IRJ. 2017;15(1):5-14. http://dx.doi.org/10.18869/nrip.irj.15.1.5.

6. Wong CL, Ching TYC, Cupples L, Button L, Leigh G, Marnane V, et al. Psychosocial development in 5-year-old children with hearing loss using hearing aids or cochlear implants. Trends Hear. 2017;21:1-19. http://dx.doi. org/10.1177/2331216517710373. PMid:28752809.

7. Bornstein S. Factors influencing future auditory function and human development in infants with hearing loss. J Health Commun. 2018;3:1-4.

8. Ching TYC, Dillon H, Leigh G, Cupples L. Learning from the longitudinal outcomes of children with hearing impairment (LOCHI) study: summary of 5-year findings and implications. Int J Audiol. 2018;57(Suppl 2):S105-11. http://dx.doi.org/10.1080/14992027.2017.1385865. PMid:29020839.

9. Ferreira K, Moret ALM, Bevilacqua MC, Jacob RST. Translation and adaptation of functional auditory performance indicators (FAPI). J Appl Oral Sci. 2011;19(6):586-98. http://dx.doi.org/10.1590/S1678-77572011000600008. PMid:22230992.

10. Padilha RB, Deperon TM, Mendes ACA, Novaes BCAC. Speech perception: performance parameters and implications for speech therapy with hearing impaired children. Distúrb Comun. 2016;12:38-49.

11. Bradham TS, Fonnesbeck C, Toll A, Hecht BF. The listening and spoken language data repository: design and project overview. Lang Speech Hear Serv Sch. 2018;49(1):108-20. http://dx.doi.org/10.1044/2017_LSHSS-16-0087. PMid:29222559.

12. Magalhães LA, Cimonari PM, Novaes BCAC. Evaluation of speech perception in hearing impaired children: the question of instrument and its criteria. Rev Soc Bras Fonoaudiol. 2007;12:221-32.

13. Leandro FSM, Costa EC, Mendes BCA, Novaes BCAC. Hearing questionnaire: semantic and cultural adaptation of the version of the Littlears questionnaire in Portuguese in families of children with hearing loss. Audiol Commun Res. 2016;21:1-12.

14. Levy CCAC, Rodrigues-Sato LCCB. Validação do questionário Parent's Evaluation of Aural/Oral Performance of Children - PEACH em língua portuguesa brasileira. CoDAS. 2016;28(3):205-11. PMid:27253225.

15. Queiroz VB, Zamberlan-Amorim NE, Pinotti KJ, Lizzi EAS, Reis ACMB Speech perception test with pictures: applicability in children with hearing impairment. Rev CEFAC. 2017;19:465-74.

16. Farsen TC, Fiorini MC, Bardagi MP. Psychometric analysis of validated instruments in several contexts: the case of the career adaptability scale. Rev. Interinst. Psicol. 2017;10(2):162-75.

17. Echevarría-Guanilo ME, Gonçalves N, Romanoski PJ. Psychometric properties of measurement instruments: conceptual bases and evaluation methods - part I. Texto Contexto Enferm. 2017;26:1-12.

18. Souza AC, Alexandre NMC, Guirardello EB, Souza AC, Alexandre NMC, Guirardello EB. Propriedades psicométricas na avaliação de instrumentos: avaliação da confiabilidade e da validade. Epidemiol Serv Saude. 2017;26(3):649-59. http://dx.doi.org/10.5123/S1679-49742017000300022. PMid:28977189.

19. Cunha CM, Almeida OP No, Stackfleth R. Main psychometric evaluation methods of the validity of measuring instruments. Rev. Aten. Saúde. 2016;14(47):75-83. http://dx.doi.org/10.13037/rbcs.vol14n47.3391.

20. Meyer TS, Figueiredo VLM. Proposta de uma Forma Reduzida do WISCIV para Avaliação Intelectual de Surdos. Aval Psicol. 2017;16(3):310-7. http://dx.doi.org/10.15689/ap.2017.1603.12534.

21. Rabelo GRG, Melo LPF. Orientação no processo de reabilitação de crianças deficientes auditivas na perspectiva dos pais. Rev CEFAC. 2016;18(2):3628. http://dx.doi.org/10.1590/1982-0216201618212515.

22. Lima MCO, Souza AS, Santos IRD, Carvalho WLO, Brazorotto JS. Análise da efetividade de um programa de intervenção para famílias de crianças com deficiência auditiva. CoDAS. 2019;31(3):e20180116. PMid:31271580.

\section{Contribuição dos autores}

MEBA participou da revisão da Literatura, escrita do projeto de pesquisa, coleta e análise dos dados e da elaboração do manuscrito; MCOL contribuiu com a coleta e análise dos dados e elaboração do manuscrito original; WLOC realizou a análise estatística dos dados e contribuiu na revisão do manuscrito; JSB participou, na condição de orientadora, da concepção do estudo, supervisão do processo de coleta de dados, análise dos dados, elaboração e revisão do manuscrito original. 
Anexo 1. Protocolo Indicadores de Performance Funcional Auditiva - Versão Reduzida

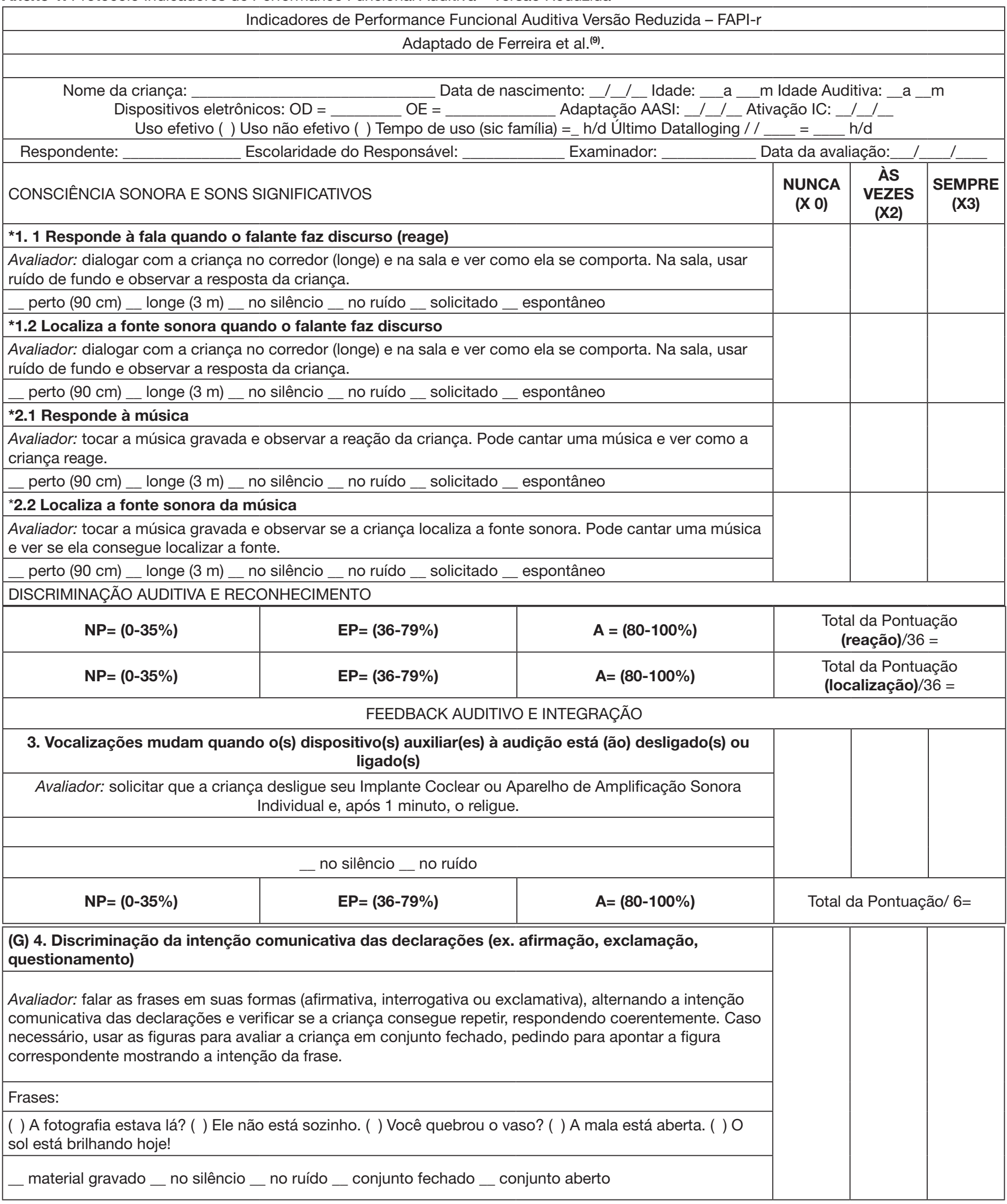

Nota 1: NP - não presente, EP - em processo, A - adquirido; Nota 2: *Itens que deverão ser aplicados no corredor (situação de distância) e na sala (situação de ruído), de forma controlada; Nota 3: (G) Itens que, inicialmente, devem ser testados com material gravado. Caso o avaliador note dificuldade da criança em escutar a mensagem gravada, proceder com a aplicação do item na condição viva-voz. 
Anexo 1. Continuação...

\section{(G) 5. Discriminação das declarações orais - palavras verdadeiras}

Avaliador: primeiro solicitar que a criança repita as onomatopeias. Caso ela não consiga, fazer a tarefa de discriminação, emitindo dois sons iguais e um diferente, observando se a criança reage alterando a atenção (para crianças pequenas) quando a palavra diferente for dita. Também pode ser feito com o apoio de figuras, com o mesmo procedimento, solicitando que a criança pegue ou aponte para a figura do som diferente.

Onomatopeias - reconhecimento em conjunto aberto:

( ) Cocorocó ( ) Quack ( ) Cri cri cri ( ) Miau ( ) Oinc oinc

Onomatopeias - discriminação:

( ) Cocorocó x cocorocó x boom ( ) Cri cri cri x cri cri cri x toc toc ( ) Miau x miau x chuá chuá

_ material gravado _ no silêncio _ no ruído _ conjunto fechado _ conjunto aberto

\section{*6. Reconhecimento do nome próprio da criança}

Avaliador: pode, ainda no corredor, chamar a criança pelo nome e observar como ela reage. Fazê-lo também próximo à criança, em situação de ruído.

_ longe (3 m) _ no silêncio _ no ruído

(G) 7. Palavras familiares baseadas em diferentes consoantes (ex. pão/mão, dar/mar, céu/mel, chão/ mão, rua/lua, pia/tia).

Avaliador: solicitar que a criança repita palavras familiares monossilábicas baseadas em diferentes consoantes. Caso não consiga, pedir que a criança as identifique em conjunto fechado.

Lista de Palavras:

( ) Pão ( ) Mão ( ) Rua ( ) Lua ( ) Céu ( ) Mel

_ material gravado _ no silêncio _ no ruído _ conjunto fechado _ conjunto aberto

\begin{tabular}{|l|l|l|c|}
\hline$N P=(0-35 \%)$ & $E P=(36-79 \%)$ & $A=(80-100 \%)$ & Total da Pontuação/54= \\
\hline
\end{tabular}

\section{RECONHECIMENTO E COMPREENSÃO AUDITIVA}

(G) 8. Identifica figura ou objeto com um elemento crítico (exemplo: aponte o carro)

Avaliador: falar as frases solicitando que a criança aponte para a figura correspondente.

( ) Aponte a moto ( ) Aponte o navio ( ) Aponte o carro ( ) Aponte o braço ( ) Aponte a perna

( ) Aponte o nariz

_ material gravado _ no silêncio _ no ruído

(G) 9. Identifica figuras ou objetos com dois elementos críticos

Avaliador: falar as frases solicitando que a criança aponte para a figura correspondente.

( ) Aponte o carro verde ( ) Aponte o avião vermelho ( ) Aponte o caminhão azul

( ) Aponte o cachorro marrom ( ) Aponte o gato amarelo ( ) Aponte o pato grande

_ material gravado _ no silêncio _ no ruído

(G) 10. Identifica figuras ou objetos com três elementos críticos

Avaliador: falar as frases solicitando que a criança aponte para a figura correspondente.

( ) Aponte o carro verde que está em cima da mesa

( ) Aponte o carro vermelho que está embaixo da mesa

( ) Aponte os olhos da menina que usa roupa vermelha

( ) Aponte a roda do carro azul grande

( ) Aponte o pé da menina que tem cabelo preto

( ) Aponte a barriga da menina que usa roupa azul

_ material gravado _ no silêncio _ no ruído

\section{(G) 11. 1 Identifica elementos críticos em pequenas histórias}

Avaliador: passar o material gravado de uma história infantil, ou lê-la para a criança, e realizar perguntas sobre a história ouvida, sem permitir a Leitura Orofacial.

\section{Perguntas simples:}

( ) Quem são os personagens da história? ( ) De que era feita a casa do primeiro porquinho? ( ) O que o lobo fez para derrubar a casa de palha?

_ material gravado _ no silêncio _ no ruído

Nota 1: NP - não presente, EP - em processo, A - adquirido; Nota 2: *Itens que deverão ser aplicados no corredor (situação de distância) e na sala (situação de ruído), de forma controlada; Nota 3: (G) Itens que, inicialmente, devem ser testados com material gravado. Caso o avaliador note dificuldade da criança em escutar a mensagem gravada, proceder com a aplicação do item na condição viva-voz. 
Anexo 1. Continuação...

\section{(G) 11. 2 Identifica elementos críticos em pequenas histórias}

Avaliador: passar o material gravado de uma história infantil, ou lê-la para a criança, e realizar perguntas sobre a história ouvida, sem permitir a Leitura Orofacial.

\section{Perguntas complexas:}

( ) Por que o lobo não conseguiu derrubar a casa do terceiro porquinho? ( ) O que o lobo fez para tentar entrar na casa de tijolos? ( ) Por que os porquinhos terminaram a história felizes?

_ material gravado _ no silêncio _ no ruído

\begin{tabular}{|l|l|}
\hline $\mathrm{NP}=(\mathbf{0}-35 \%)$ & $\mathrm{EP}=(\mathbf{3 6 - 7 9} \%)$ \\
\hline
\end{tabular}

MEMÓRIA AUDITIVA DE CURTO PRAZO

(G) 12.1 Memória: recorda números que foram ouvidos ou demonstrados logo após o estímulo (CRIANÇAS MAIORES)

Avaliador: solicitar que a criança repita as combinações de 2 dígitos.

( ) $9-1$ ( ) $7-9$ ( ) $3-5$ material gravado _ no silêncio _ no ruído _ com pistas visuais _ só auditivo

(G) 12.2 Memória: recorda números que foram ouvidos ou demonstrados logo após o estímulo (CRIANÇAS MAIORES)

Avaliador: solicitar que a criança repita as combinações de 3-4 dígitos.

( ) $6-4-9$ ( ) $6-3-5-1$ ( ) $8-2-9-3$

_ material gravado _ no silêncio _ no ruído _ com pistas visuais _ só auditivo

(G) 12.3 Memória: recorda números que foram ouvidos ou demonstrados logo após o estímulo (CRIANÇAS MAIORES)

Avaliador: solicitar que a criança repita as combinações de 5-6 dígitos.

( ) $4-7-3-9-9$ ( ) $2-9-6-1-8-3($ ) $6-9-5-7-2-8$

__ material gravado _ no silêncio _ no ruído _ com pistas visuais _ só auditivo

(G) 12.4 Memória: recorda números que foram ouvidos ou demonstrados logo após o estímulo (CRIANÇAS MENORES)

Avaliador: solicitar que a criança repita onomatopeias. Caso ela demonstre dificuldade, apresentar as figuras dos animais para que a criança as coloque na ordem do áudio ou da fala da terapeuta.

( ) Au au ( ) Au au, miau ( ) Moo, quack, bée ( ) Au au, miau, bée, quack ( ) Moo, quack, bée, au au, miau __ material gravado _ no silêncio _ no ruído _ com pistas visuais _ só auditivo

\begin{tabular}{|c|c|c|}
\hline$N P=(0-35 \%)$ & $E P=(36-79 \%)$ & $A=(80-100 \%)$ \\
\hline$N P=(0-35 \%)$ & $E P=(36-79 \%)$ & $A=(80-100 \%)$ \\
\hline
\end{tabular}

\section{PROCESSAMENTO AUDITIVO LINGUÍSTICO}

*13.1 (CRIANÇAS MENORES). Aplicação da informação auditiva: criança compreende e utiliza informação auditiva e retira do seu conhecimento geral o significado de uma variedade de situações:

Avaliador: observar o comportamento vocal/troca de turnos, propondo uma situação de diálogo com a criança, enquanto ela realiza alguma atividade simultânea.

Proposta de materiais para a atividade simultânea: livro de pintura, lápis de cor ou brinquedos distrativos, como quebra-cabeça/torremoto.

Possíveis perguntas: Qual é a sua brincadeira favorita? Quais são os desenhos que você gosta de assistir? Como é o nome dos seus amiguinhos? (Estas questões são sugestões. O fonoaudiólogo poderá substituir e acrescentar de acordo com a necessidade e aplicação com cada criança).

_ no silêncio _ no ruído

Nota 1: NP - não presente, EP - em processo, A - adquirido; Nota 2: *Itens que deverão ser aplicados no corredor (situação de distância) e na sala (situação de ruído), de forma controlada; Nota 3: (G) Itens que, inicialmente, devem ser testados com material gravado. Caso o avaliador note dificuldade da criança em escutar a mensagem gravada, proceder com a aplicação do item na condição viva-voz. 
Anexo 1. Continuação...

13.2 (CRIANÇAS MAIORES). Aplicação da informação auditiva: criança compreende e utiliza informação auditiva e retira do seu conhecimento geral o significado de uma variedade de situações (conversação):

Avaliador: enquanto a criança realiza uma atividade, conversar com ela utilizando diversos tipos de questionamentos.

Proposta de materiais para a atividade simultânea: livro de pintura, lápis de cor ou brinquedos distrativos, como quebra-cabeça/torremoto.

Possíveis perguntas: Quem veio com você para a terapia? Para quais lugares você gosta de ir? O que você gosta de fazer nos finais de semana? (Estas questões são sugestões. O fonoaudiólogo poderá substituir e acrescentar de acordo com a necessidade e aplicação com cada criança).

_ com pistas visuais _ só auditivo _ no silêncio _ no ruído _ atividade sozinha _ atividades simultâneas

\section{Conversa por telefone}

Avaliador: enquanto realiza uma atividade, solicitar que o familiar saia da sala e fale com a criança pelo telefone.

Sugestões de perguntas para o familiar fazer: O que você está fazendo? Qual o nome da sua fono? Está gostando da atividade? O que você vai fazer mais tarde?

(Estas questões são sugestões. O fonoaudiólogo poderá substituir e acrescentar de acordo com a necessidade e aplicação com cada criança).

_ atividade sozinha _ atividades simultâneas

(G) 15. 1 (PARA CRIANÇAS MENORES): segue uma instrução

Avaliador: solicitar que a criança realize algumas ações.

( ) Bata palmas ( ) Pegue na cabeça ( ) Bata os pés no chão

_ material gravado _ ruído _ silêncio

(G) 15. 2 (PARA CRIANÇAS MENORES): seguem duas instruções

Avaliador: solicitar que a criança realize algumas ações.

Observação: deixar figuras de diferentes categorias semânticas ao alcance da criança, dentre elas a de um animal. Sugestões de figuras: cachorro, mesa, navio e menina.

( ) Pegue a figura de um animal e a coloque embaixo da mesa

( ) Mande um beijo e dê tchau

( ) Pegue no nariz e depois no olho

_ material gravado _ ruído _ silêncio

(G) 15. 3 (PARA CRIANÇAS MENORES): seguem três instruções

Avaliador: solicitar que a criança realize algumas ações.

Observação: deixar figuras de diferentes categorias semânticas ao alcance da criança, dentre elas a de um animal. Sugestões de figuras: cachorro, mesa, navio e menina.

( ) Toque a ponta do nariz, bata palmas e pegue uma figura

( ) Mostre a língua, toque na barriga e bata os pés no chão

( ) Pegue no cabelo, fique em pé e ande pela sala

_ material gravado __ ruído _ silêncio

(G) 15.4 (PARA CRIANÇAS MAIORES): seguem ordens e instruções

Avaliador: solicitar que a criança realize algumas ações.

( ) Pegue no cabelo e fique em pé

( ) Sorria, dê tchau e mande um beijo

( ) Faça uma careta, finja que está dormindo e estire a língua

_ material gravado _ ruído _ silêncio

\begin{tabular}{|l|l|l|c|}
\hline $\mathrm{NP}=(\mathbf{0}-\mathbf{3 5 \% )}$ & $\mathrm{EP}=\mathbf{( 3 6 - 7 9 \% )}$ & $\mathrm{A}=\mathbf{( 8 0 - 1 0 0 \% )}$ & $\begin{array}{c}\text { Total da Pontuação (crianças } \\
\text { menores)/39= }\end{array}$ \\
\hline $\mathrm{NP}=\mathbf{( 0 - 3 5 \% )}$ & $\mathrm{EP}=\mathbf{( 3 6 - 7 9 \% )}$ & $\mathrm{A}=\mathbf{( 8 0 - 1 0 0 \% )}$ & $\begin{array}{c}\text { Total da Pontuação (crianças } \\
\text { maiores)/33 }=\end{array}$ \\
\hline
\end{tabular}

Nota 1: NP - não presente, EP - em processo, A - adquirido; Nota 2: *Itens que deverão ser aplicados no corredor (situação de distância) e na sala (situação de ruído), de forma controlada; Nota 3: (G) Itens que, inicialmente, devem ser testados com material gravado. Caso o avaliador note dificuldade da criança em escutar a mensagem gravada, proceder com a aplicação do item na condição viva-voz. 